\title{
NOTE ON THE EXPANSION OF ANALYTIC FUNCTIONS IN SERIES OF POLYNOMIALS AND IN SERIES OF OTHER ANALYTIC FUNCTIONS*
}

BY

\author{
J. L. WALSH
}

On several occasions $\dagger$ the present writer has studied the expansion of functions of a complex variable in series of more or less arbitrary functions. The results obtained are connected with Birkhoff's generalization of Taylor's series, $\ddagger$ but yield additional results when interpreted, after conformal transformation of the plane, as the expansion of arbitrary functions in series of polynomials. The object of the present note is to prove the following theorem, which is easily established by the methods already used, but seems to go in some directions beyond results proved by other writers§ concerning polynomials associated with a region bounded by an arbitrary Jordan curve:

In the plane of the complex variable $z$ let $C$ be an arbitrary Jordan curve in whose interior the origin lies. Then there exists a set $\left\{p_{k}(z)\right\}$ of polynomials in $z$ having respectively $k$-fold roots at the origin and no other roots in or on $C$, and a set of functions $\left\{s_{k}(z)\right\}$, each a polynomial in $1 / z$ of degree $k+1$, and such that

$$
\int_{C^{\prime}} s_{k}(z) p_{i}(z) d z= \begin{cases}0, & i \neq k, \\ 1, & i=k,\end{cases}
$$

where the integral is taken in the positive sense over an arbitrary rectifiable Jordan curve $C^{\prime}$ interior to $C$ but which contains the origin in its interior.

* Presented to the Society, September 7, 1928; received by the editors in July, 1928.

† These Transactions, vol. 22 (1921), pp. 230-239; vol. 26 (1924), pp. 155-170; vol. 30 (1928), pp. 307-332. We shall refer to these papers as I, II, III respectively.

‡ Paris Comptes Rendus, vol. 164 (1917), pp. 942-945. A number of later writers have studied the same subject, under somewhat different hypotheses from those of Birkhoff. Detailed references are given by Widder, in the present number of these Transactions, pp. 43-52.

$\S$ References to Faber and Szegö are given in II; see also Fejér, Interpolation und konforme Abbildung, Göttinger Nachrichten, 1918, pp. 319-331, and Carleman, Über die Approximation analytischer Funktionen, Arkiv för Matematik, Astronomi och Fyșik, vol. 17 (1922-23). Fejér considers the development of functions analytic in the closed regions, Carleman of functions analytic interior to the given regions, integrable and with an integrable square (in the sense of surface integral) over the closed regions.

For developments in functions more general than polynomials, see Bergmann, Mathematische Annalen, vol. 86 (1922), pp. 238-271; Bochner, Mathematische Zeitschrift, vol. 14 (1922), pp. 180-207. 
If $F(z)$ is an arbitrary function analytic interior to $C_{2}$, then the series

$$
\sum_{k=0}^{\infty} a_{k} p_{k}(z), \quad a_{k}=\int_{C^{\prime}} F(z) s_{k}(z) d z,
$$

converges to the sum $F(z)$ throughout the interior of $C$; the convergence is uniform in any closed region interior to $C$. The coefficients $a_{k}$ in (1) can also be written in the form

$$
a_{k}=A_{0}{ }^{(k)} F(0)+A_{1}{ }^{(k)} F^{\prime}(0)+\cdots+A_{k}{ }^{(k)} F^{(k)}(0),
$$

where $A_{i}^{(k)}$ is a constant independent of $F(z)$, and where $F^{(i)}(0)$ indicates the ith derivative of $F(z)$ at the origin.

The interior of $C$ can be mapped one-to-one and conformally on the interior of the unit circle $\gamma$ in the $x$-plane by some transformation $x=\phi(z), z=\psi(x)$, where $\phi(0)=0$, and the transformation is continuous in the closed regions involved. In general we denote by $C_{\rho}$ the transform of the circle $|x|=\rho$, where $0<\rho<1$.

If $F(z)$ is an arbitrary function analytic interior to $C_{\rho}$, continuous in the corresponding closed region, and satisfies a Lipschitz condivion on $C_{\rho}$, then the series (1), where $C^{\prime}$ is interior to $C_{\rho}$, converges uniformly in this closed region to the value $F(z)$. If $F(z)$ is required merely to be analytic interior to $C_{\rho}$, and is continuous in the corresponding closed region, then (1) converges uniformly, when summed by the method of Cesiro, to the value $F(z)$ in the closed region bounded by $C_{\rho}$. If $F(z)$ is subjected to no other condition than that of being analytic interior to $C_{\rho}$, series (1) converges interior to $C_{\rho}$ to the sum $F(z)$; the convergence is uniform in any closed region interior to $C_{\rho}$.

If $F(z)$ is an arbitrary function defined on $C_{\rho}$, integrable in the sense of Lebesgue, and if the condition*

is satisfied, then the two series

$$
\int_{C_{\rho}} F(z) z^{k} d z=0 \quad(k=0,1,2, \cdots),
$$

$$
\begin{array}{ll}
\sum_{k=0}^{\infty} a_{k} p_{k}[\psi(x)], & a_{k}=\int_{|x|-\rho} F[\psi(x)] s_{k}[\psi(x)] \psi^{\prime}(x) d x, \\
\sum_{k=0}^{\infty} a_{k}^{\prime} x^{k}, & a_{k}^{\prime}=\frac{1}{2 \pi i} \int_{|x|=\rho} F[\psi(x)] x^{-k-1} d x,
\end{array}
$$

* As in III, Theorem IX, no condition is here necessary if we set

instead of (2).

$$
a_{k}=\int_{|x|-\infty} F[\psi(x)] P_{k}(x) d x
$$


have essentially the same convergence properties on and within the circle $|x|=\rho$, in the sense that in this closed region their term-by-term difference converges absolutely and uniformly to the sum zero.

An arbitrary series of the form

$$
\sum_{k=0}^{\infty} g_{k} p_{k}(z)
$$

which converges for a single point $z$ on $C_{\rho}$, converges uniformly interior to $C_{\rho^{\prime}}$, if $\rho^{\prime}<\rho$. If (3) diverges for a point $z$ on $C_{\rho}$, that series diverges for all points $z$ exterior to $C_{\rho}$ and on or interior to $C$. If in general we set

$$
\limsup _{k \rightarrow \infty}\left|g_{k}\right|^{1 / k}=\frac{1}{\rho},
$$

then if $\rho \leqq 1$, series (3) converges for $z$ interior to $C_{\rho}$ and diverges for $z$ exterior to $C_{\rho}$ bui on or interior to $C$; if $\rho>1$, series (3) converges for $z$ on or interior to $C$. If $0<\rho<1$, some singular point of the function represented by the series lies on the curve $C_{\rho}$.

If (3) converges for the value $z=z_{1}$ on $C_{\rho}$, then that series converges uniformly in the closed region bounded by two arbitrary line segments whose interiors are interior to $C_{\rho}$ and which terminate in $z_{1}$, and by an arc of a curve $C_{\rho^{\prime}}$, where $\rho^{\prime}<\rho$. If (3) converges uniformly on an arc $z_{1} z_{2}$ of the curve $C_{\rho}$, then that series converges uniformly in the closed region bounded by that arc, by two arbitrary line segments whose interiors are interior to $C_{\rho}$ and which terminate respectively in $z_{1}$ and $z_{2}$, and by an arc of the curve $C_{\rho^{\prime}}$, where $\rho^{\prime}<\rho$.

If (3) is such that $\lim _{k \rightarrow \infty} k g_{k} \rho^{i k}=0$, and if for approach along the normal ${ }^{*}$ to $C_{\rho}$ to the point $z_{1}$ on $C_{\rho}$ we have

$$
\lim _{z \rightarrow z_{1}} f(z)=g,
$$

where $f(z)$ denotes the value of the series (3), which is convergent, under the hypothesis, for $z$ interior to $C_{p}$, then we have also

$$
\sum_{k=0}^{\infty} g_{k} p_{k}\left(z_{1}\right)=g .
$$

An arbitrary series (3), convergent for a single value of $z$ interior to $C$ but not the origin, is the unique expansion of form (1) of some function $F(z)$ analytic on and within some curve $C_{\rho}$.

* A more general theorem might easily be stated; see III, p. 313, third footnote. In III, Theorem VI, for $g_{k} / k$ read $k g_{k}$, and in III, Theorem IX, for $g_{k}\left(k \rho^{k}\right)^{-1}$ read $k g_{k} \rho^{k}$. 
Almost this entire theorem results from the two facts that the functions $\phi(z)$ and $\psi(x)$ are continuous in the closed interiors of $C$ and $\gamma$ respectively, and that in the former closed region $\phi(z)$ or any power of it can therefore be uniformly approximated as closely as desired by means of a polynomial in $z$; this approximating polynomial can even be chosen so that it has a zero at the origin whose multiplicity is the power of $\phi(z)$. For the function $[\phi(z) / z]^{k}$ is analytic interior to $C$, continuous in the corresponding closed region, hence in that region can be approximated as closely as desired by a polynomial in $z$. This gives directly the polynomial $p_{k}(z)$ (as in II, p. 163, and III, p. 316) which has a $k$-fold root at the origin and is such that

$$
\left|p_{k}(z)-[\phi(z)]^{k}\right| \leqq \epsilon_{k}
$$

is satisfied for all $z$ on or interior to $C$. Inequality (4) implies, by virtue of III, $\S 6$, that the polynomials $p_{k}(z)$ just considered satisfy the requirements of III, Theorem IX, where an arbitrary curve $C_{\rho}$ of the present theorem plays the rôle of the curve $C$ of III. The theorem as stated follows, then, with the exception of a minor point about to be mentioned, because the same set of polynomials $p_{k}(z)$ can be considered independent of $\rho$ for any curve $C_{\rho}$.

In II and III there were considered as given functions for expansion not arbitrary functions integrable in the sense of Lebesgue, but merely functions integrable and with an integrable square. The more general functions might easily have been treated there, however, as we shall now see by reference to I.

We assume the series $\sum \epsilon_{n}$ to be dominated by a convergent geometric series, $\epsilon_{n} \leqq R \tau^{n}, \tau<1$, so we have by III, p. 309, inequality (8),

$$
\left|c_{n k}\right| \leqq \frac{R \tau^{n}}{(1+\epsilon)^{k}}
$$

In the notation of $I{ }^{*}$ we have also (I, p. 234, (13) and (11))

$$
\left|c_{k i}+\bar{d}_{i k}\right| \leqq \frac{\gamma_{i} p_{k}}{1-p}, \quad \text { where } \gamma_{i}^{2}=\sum_{j=0}^{\infty}\left|c_{j i}\right|^{2}, \quad p_{k}^{2}=\sum_{j=0}^{\infty}\left|c_{k j}\right|^{2}
$$

Then the two double series $\sum_{n, k=0}^{\infty}\left|c_{n k}\right| ; \sum_{i, k=0}^{\infty}\left|d_{i k}\right|$ are absolutely convergent. The formal work of I, pp. 235-236, can now be justified immediately. If (in the notation of I) the function $f(x)$ is integrable in the sense of Lebesgue, the coefficients $a_{k}$ are bounded in their totality; in fact a well known theorem due to Lebesgue asserts that $\lim _{k \rightarrow \infty} a_{k}=0$. The convergence of the

\footnotetext{
* Slightly altered, for in I we consider only real functions, but in II and III complex functions as well. Compare II, p. 160.

The present inequalities replace those of III, p. 309, (10), (11), in which $d_{k i}$ should be replaced by $\bar{d}_{k i}$, and $p_{k}$ by $\gamma_{k}$.
} 
series in the right-hand member of (16) (I, p. 236) follows now at once, for a dominant convergent series of positive terms exists.

This proves, then, that in III, Theorem I, the two series (3) and (4) have for $|x| \leqq 1$ essentially the same convergence properties, provided merely that $F(x)$ is integrable on $\gamma$ in the sense of Lebesgue. The theorem of the present paper contains an application of this result.

The present theorem suggests, and in fact is easily proved by means of a simple modification of III, Theorems I-VIII. This modification, which we do not present in detail, replaces the requirement of III, Theorem I, that the functions $p_{k}(x)$ should be analytic for $|x| \leqq 1+\epsilon$, by the requirement that these functions should be continuous for $|x| \leqq 1$, analytic for $|x|<1$. Expansions in terms of the functions $p_{k}(x)$ are then studied only for $|x| \leqq 1$, and the equivalence of expansions of arbitrary functions is considered, so far as the present methods apply without modification, only on circles $|x|=\rho<1$.

In the theorem of the present note, the convergence of series (1) and (3) on the boundary of $C$ deserves special study, with particular reference to the nature of the curve $C$ (rectifiability, existence of tangents, etc.), although if $C$ itself is analytic, a fairly complete study is of course contained in III. We make one further elementary remark in this connection. If $C$ possesses forward and backward tangents at a point $z_{1}$, the transformation $x=\phi(z)$ is quasi-conformal at that point.* It follows, then, from the proof given in III, pp. 311, 312, that if (3) (notation of the present paper) converges for a point $z_{1}$ of $C$, and if $C$ possesses forward and backward tangents at $z_{1}$, then (3) converges uniformly in the closed region bounded by two arbitrary line segments interior to $C$ but not tangent to $C$ terminating in $z_{1}$, and by an arc of an arbitrary curve $C_{\rho}, \rho<1$.

Similarly, if (3) converges uniformly on an $\operatorname{arc}$ of $C$ bounded by $z_{1}$ and $z_{2}$, and if $C$ possesses forward and backward tangents at $z_{1}$ and $z_{2}$, then (3) converges uniformly in the closed region bounded by two arbitrary line segments interior to $C$ but not tangent to $C$, terminating in $z_{1}$ and $z_{2}$ respectively, and by an arc of an arbitrary curve $C_{\rho}$.

* Carathéodory, Schwarz Festschrift, 1914, pp. 19-41, §20.

HARVARD UNIVERSITY

Cambridge, Mass. 